\title{
Bibliometric analysis of recent research on 5-Fluorouracil (2015-2020)
}

\author{
Howard Ramirez-Malule ${ }^{*}$ iD , Wilson Cardona-G ${ }^{2}$ \\ ${ }^{1}$ Escuela de Ingeniería Química, Universidad del Valle, A.A. 25360, Cali 76001, Colombia. \\ ${ }^{2}$ Química de Plantas Colombianas, Institute of Chemistry, Faculty of Exact and Natural Sciences, University of Antioquia UdeA, Calle 70 No. $52-21$, A.A \\ 1226 Medellín, Colombia.
}

\begin{tabular}{l}
\hline ARTICLE INFO \\
\hline Received on: $27 / 05 / 2021$ \\
Accepted 0n: 19/09/2021 \\
Available Online: 05/01/2022
\end{tabular}

Key words:

5-Fluorouracil, anticancer

activity, bibliometric analysis.

\begin{abstract}
Cancer is one of the most widespread diseases in the world and causes millions of deaths every year. Currently, there are several anticancer treatments to deal with the different types of cancer reported around the world. However, most of them have both side effects and low selectivity to malignant cells. 5-Fluorouracil (5-FU) is used as firstline treatment against several types of cancer. Recently, hybrid and derivative molecules based on 5-FU have been reported as anticancer with high/modest activity, leading to a new and emerging therapy to treat cancer. The aim of this study was to carry out a bibliometric analysis of studies related to 5-FU in the field of chemistry. Data collection was carried out using the Scopus database, and VOSviewer 1.6.13 was used for data analysis and visualization. "5-Fluorouracil" was the term used in the search during a time span of 2015-2020. A total of 1,025 documents related to 5-FU were indexed in Scopus between 2015 and 2020, both included. Articles and reviews related to 5-FU have increased during the period of 2015-2020, starting and ending with 137 and 241 studies, respectively. The main areas, excluding chemistry, were (i) Biochemistry, Genetics, and Molecular Biology; (ii) Chemical Engineering; and (iii) Materials Science. Within these areas, the studies were focused on drug delivery, synthesis of anticancer compounds and their activity, molecular simulation, treatment of cancer cells, and drug resistance. Finally, the most productive countries on studies related to 5-FU, which also had the most robust collaboration network, were China, India, and the United States.
\end{abstract}

\section{INTRODUCTION}

Cancer disease is one of the primary public burdens and is the second leading cause of death worldwide, with 10 million deaths per year and $70 \%$ of them occuring in low-middle-income countries. In 2020, the most common causes of cancer deaths were lung, colon and rectum, liver, stomach, and breast with 1.8, 0.935, $0.830,0.769$, and 0.685 million cases, respectively. In terms of new cases of cancer, and also for 2020, the most common were breast, lung, colon and rectum, prostate, skin (nonmelanoma), and stomach with 2.26, 2.21, 1.93, 1.41, 1.20, and 1.09 million cases, respectively (WHO, 2020). In 2018, 9.6 million people globally

\footnotetext{
${ }^{*}$ Corresponding Author

Howard Ramirez-Malule, Escuela de Ingeniería Química, Universidad del Valle, A.A. 25360, Cali 76001, Colombia.

E-mail: howard.ramirez@,correounivalle.edu.co
}

were estimated to have died from cancer (WHO, 2020). Recently, global cancer statistics including incidence and mortality for 36 types of cancer in 195 countries was presented (Sung et al., 2021). Probably because of the high rate of deaths caused by cancer, many researchers have focused on cancer treatment for decades, applying different methodologies with diverse findings. Thus, new alternative therapies against cancer have emerged using compounds that exhibit good anticancer activity. Among those compounds, hybrids and conjugates based on 5-Fluorouracil (5-FU) are highlighted (see review of Cardona-G et al., 2021).

Since the growth and number of studies available on a specific topic, such as cancer, can be considerably high, the task of structuring a comprehensible review with relevant information could become a challenge for researchers. However, bibliometric analysis allows for scientific mapping of large amounts scientific literature, which could provide macroscopic overview of a topic of interest such as antibiotics, emerging contaminant, antivirals, and multidrug-resistant fungus (Gómez-Ríos, López-agudelo, 
and Ramírez-Malule, 2020; Gómez-Ríos and Ramírez-Malule, 2019; Liao et al., 2018; Natalia et al., 2021; Ramírez-Malule, 2018; Ramírez-Malule et al., 2020; Ramírez-Malule et al., 2020; Soosaraei et al., 2018). In the case of cancer treatment, there are several reviews available on cancer of breast (Waks and Winer, 2019), prostate (Nevedomskaya et al., 2018), colon (Banerjee et al., 2017), and even survivorship statistics for different cancers (Miller et al., 2016, 2019). Besides, a recent review based on 5-FU was published (Cardona-G et al., 2021). In that review, the authors reported the synthesis of hybrids and derivatives containing 5-FU and their anticancer activity in different cancer cell lines. However, a bibliometric analysis of 5-FU is still lacking. Thus, the aim of this study was to identify and analyze the studies related to 5-FU in the field of chemistry.

\section{METHODS}

\section{Database selection}

The Scopus database was used as a tool to retrieve the most relevant documents related to 5-FU in the field of "chemistry." Data collection was acquired from Scopus on March 2, 2021, and comprised records obtained from a systematic search of documents matching the search terms in the fields of article title, abstract, and keywords. The search was refined to article and review as the document types. The resulting search was as follows:

- Search equation: TITLE-ABS-KEY ("5-Fluorouracil”) AND (LIMIT-TO (DOCTYPE, “ar”) OR LIMIT-TO (DOCTYPE, "re") AND (LIMIT-TO (SUBJAREA, "CHEM").

- Timespan: 2015-2020. 1,025 articles and reviews were retrieved after removing duplicates.

\section{Data export and analysis}

The information retrieved from the Scopus database for all records during the search were (i) both citation and bibliographical information, and (ii) abstract and keywords. Retrieved data were downloaded from Scopus and exported to Microsoft Excel ${ }^{\circledR}$. Later, VOSviewer 1.6.13 was used for visualization and data analysis. Six was set as the minimum number of occurrences of a keyword. After removing the thesaurus terms, 52 of them met the threshold, which led to 9 clusters.

\section{Bibliometric indicators}

The following bibliometric indicators were evaluated:

- Volume and growth of publications related to 5-FU studies.

- Subject areas related to 5-FU studies.

- Co-occurring keywords of network visualization.

- Co-occurrening keywords of overlay visualization.

- Most active countries.

\section{Results}

A total of 1,025 documents related to 5 -FU in the field of chemistry were indexed in the Scopus database between 2015 and 2020, both included. Figure 1 shows the evolution of the number of publications in this field. As observed, there is an increase in research outputs starting and ending with 137 and 241 records for 2015-2020, respectively. In this time range, the areas

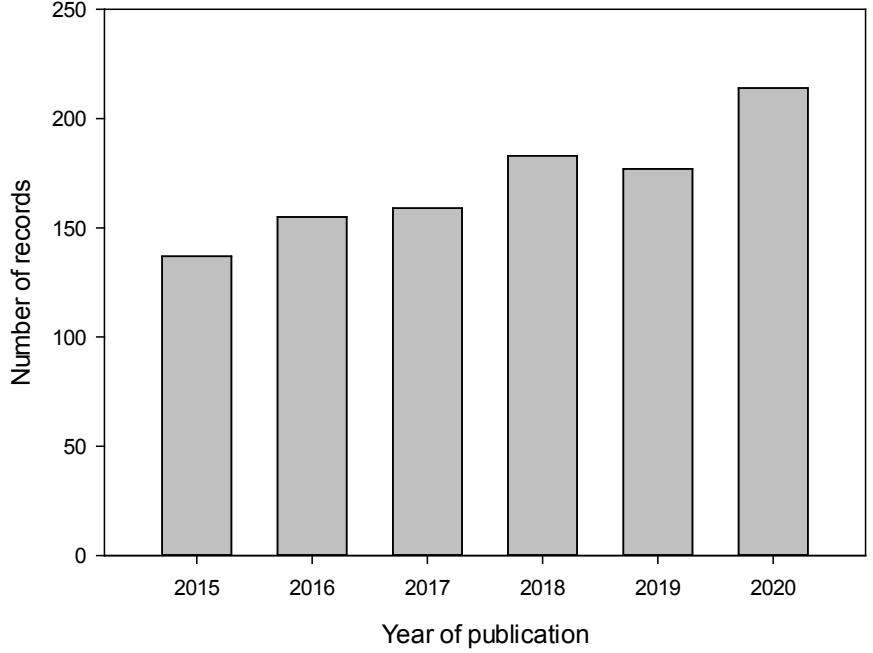

Figure 1. Evolution of published studies related to 5-FU between 2015 and 2020 .

of knowledge (excluding chemistry) related to 5-FU studies were (i) Biochemistry, Genetics, and Molecular Biology; (ii) Chemical Engineering; and (iii) Materials Science (Fig 2).

Figure 3a shows the co-occurrence of authors' keywords network, which contains nine clusters easily distinguishable by colors. This figure clearly displays the topic on which the researchers have focused in the last 6 years (2015-2020): (i) drug delivery (blue and purple); (ii) cancer treatment and drug resistance (yellow, orange, and pink); and (iii) anticancer activity (red and blue light) and molecular docking (green). Studies related to drug resistance, chemoresistance, adsorption, mesoporous

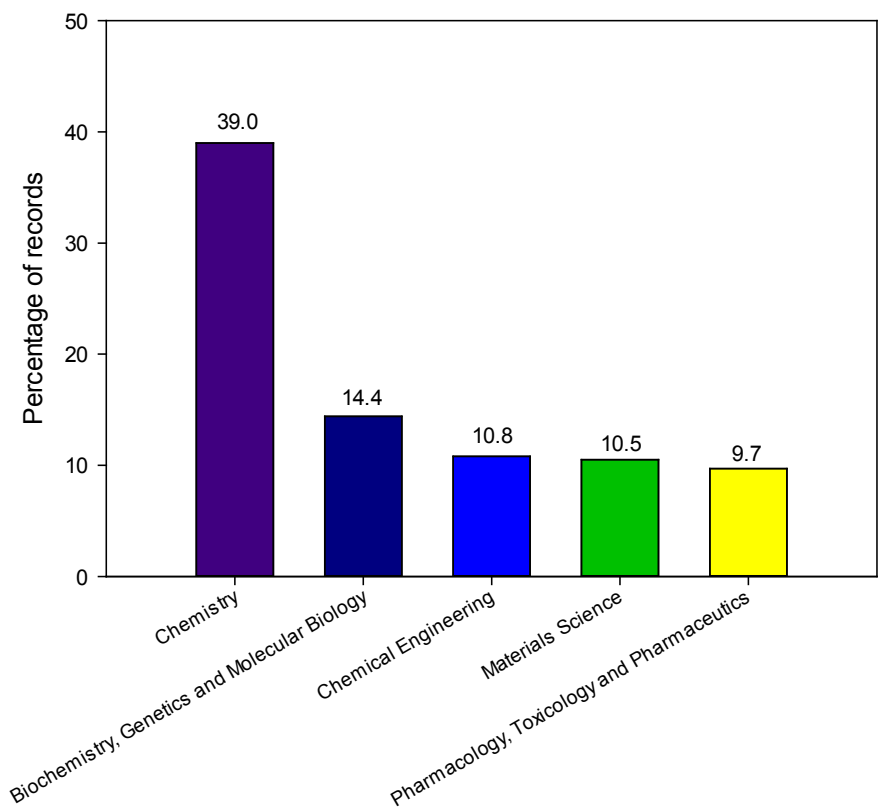

Figure 2. Summary of publications grouped by areas related to 5-FU studies between 2015 and 2020 . 
a.

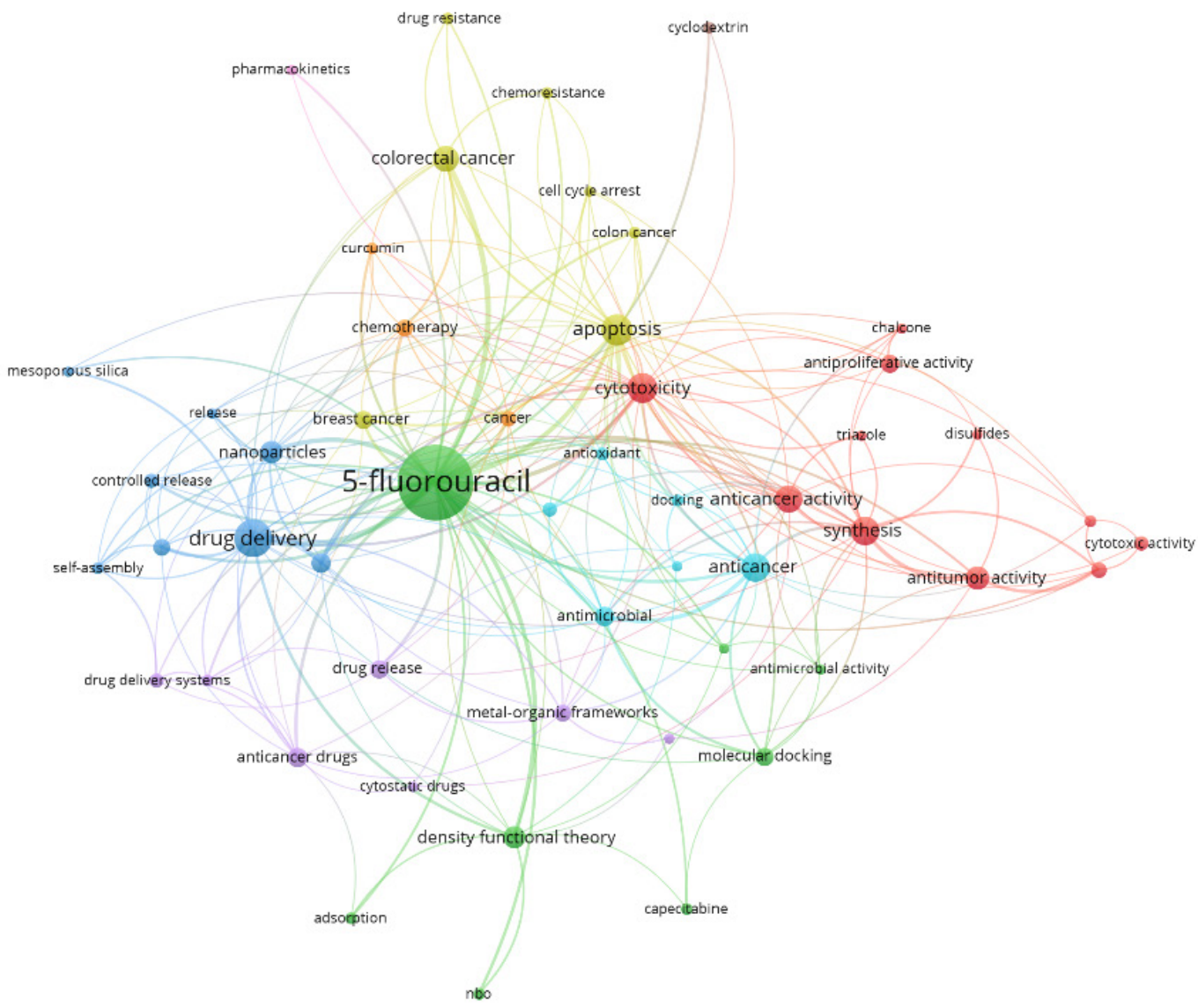

\& VOSviewer

b.

B vosviewer
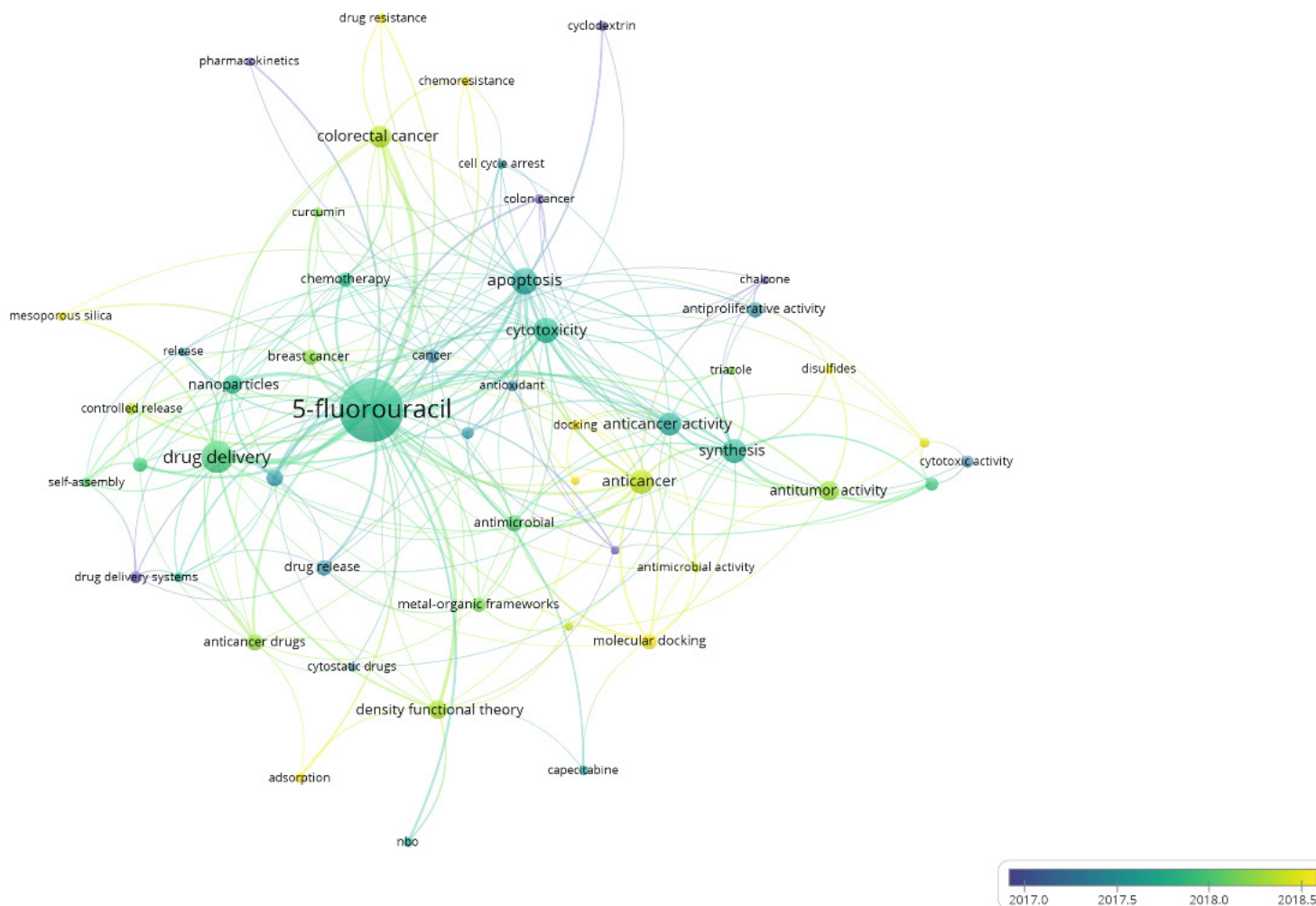

Figure 3. Bibliometric network of studies related to 5-FU between 2015 and 2020. (a) Research topic map. (b) Research topic map with time overlap. Note: minimum number of occurrences of a keyword is six. 
a.

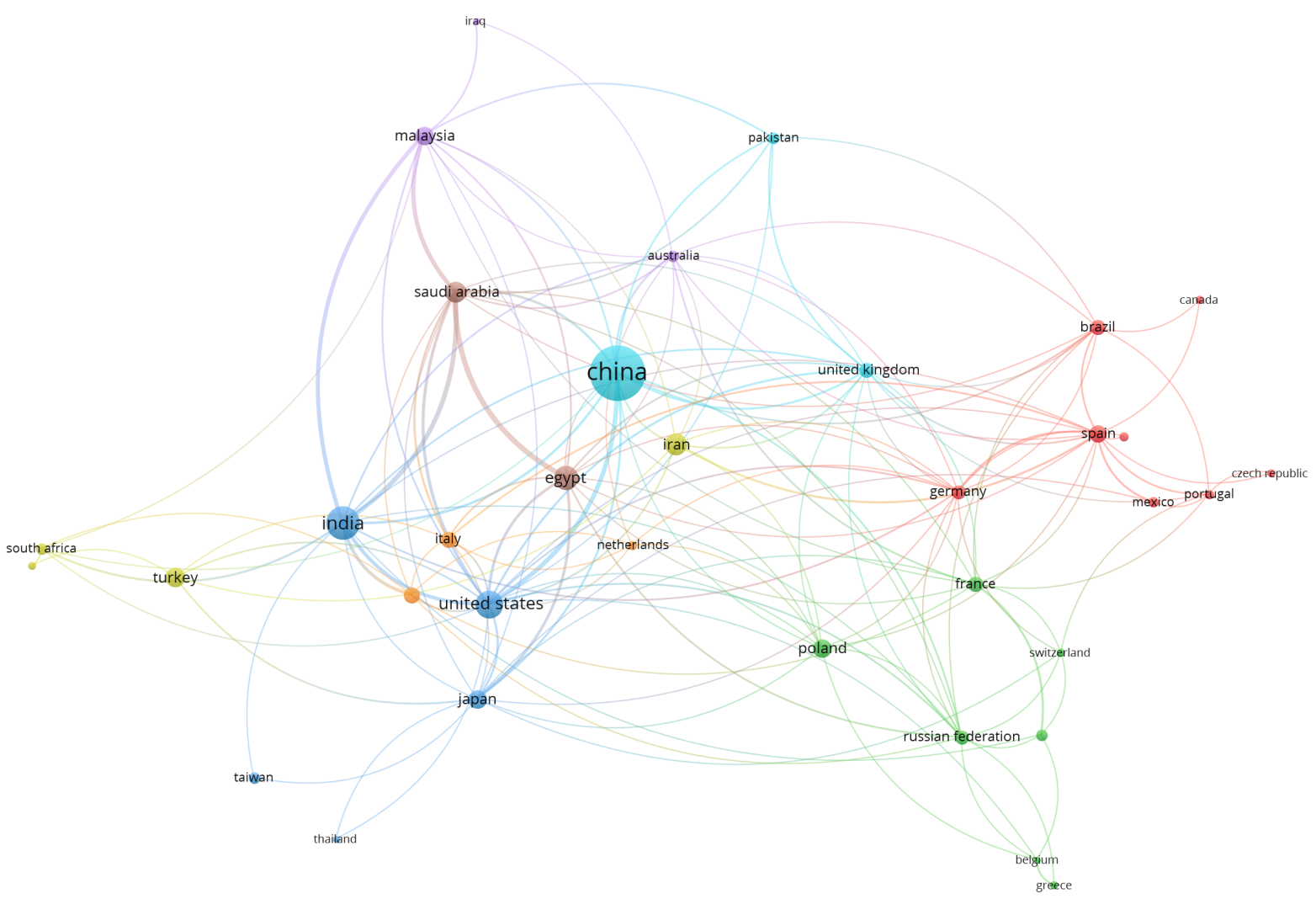

\& VOSviewer

b.

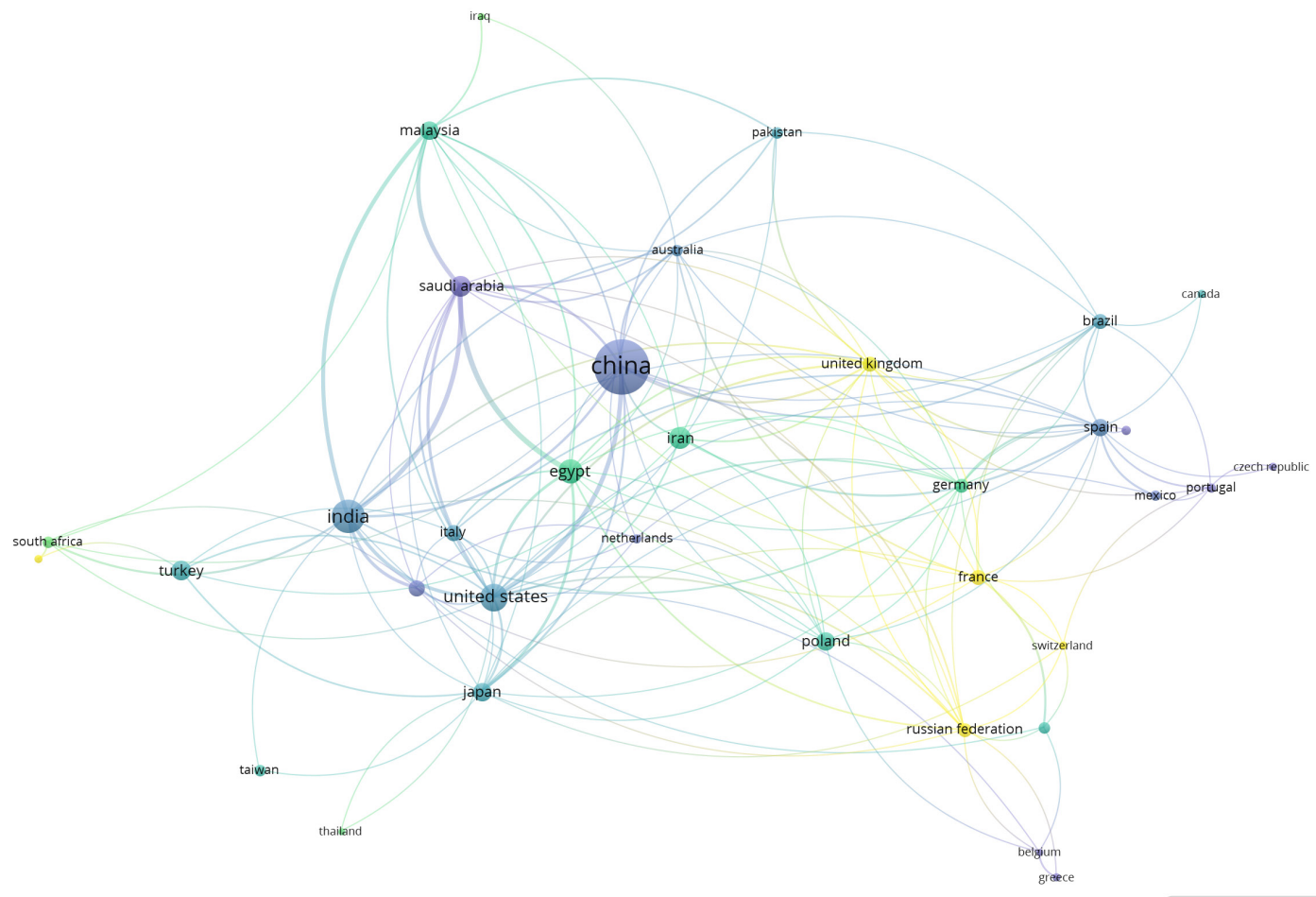

f

Figure 4. Countries collaboration network studies related to 5-FU between 2015 and 2020. (a) Network visualization. b) Overlay visualization. Note: countries contributing with a minimum number of both documents and citations of five. 
silica, and molecular docking concentrated the research on 5-FU in 2018 (Fig 3b).

In the collaboration networks of countries researching on 5-FU — in the field of chemistry - and during 2015-2020, China ranked first, followed by India and the United States with 330, 121, and 80 records, respectively (Fig 4a). These countries also have the strongest collaboration network. Nevertheless, countries such as the United Kingdom, France, Switzerland, Germany, Poland, Egypt, and Malaysia concentrated the studies between 2017 and 2018 (Fig 4b).

\section{DISCUSSION}

\section{Anticancer activity of hybrids and derivatives based on 5-FU}

5-FU (Fig 5) is an antimetabolite of the pyrimidine analog type, which is widely used in the first-line treatment for solid tumors, including colorectal being more effective with regard to others, as reviewed by Ribeiro et al. (2016), gastrointestinal tract reviewed by Sara et al. (2018) and breast cancer (Tecza et al., 2018). Although 5-FU is effective, it produces undesirable gastrointestinal and neurological side effects, which in many cases leads to dose limitations or cessation of the anticancer therapy (Rejhová et al., 2018).

In the search to reduce these side effects of 5-FU, two optimization strategies have been proposed: (1) the incorporation of pharmacologically active compounds with anticancer activity (called hybrid molecule) and (2) the functionalization with other groups of compounds (called conjugates). For 5-FU modification and from a synthetic point of view, four important precursors of 5-FU (commonly used in the synthesis of different hybrids or conjugates) have been used: 5-FU acid, 5-FU alkyl bromide, 5-FU-alcohol, and 5-FU alkyne. However, 5-FU acid is the most used (Fig 5).

5-FU colchicine hybrids were obtained via 5-FU acid (Fig 5). These compounds exhibited good cytotoxic activity against four cancer lines cells $(R=\mathrm{SMe}: 9.5,7.8,10.2$, and $7.5 \mu \mathrm{M}$ on A2780, A-549, BEL-7402, and MCF7, respectively; $R=N$ $\left(\mathrm{C}_{2} \mathrm{H}_{5}\right) 2: 10.2,10.6,10.9$, and $9.8 \mu \mathrm{M}$ on A2780, A-549, BEL7402, and MCF7, respectively) (Shen et al., 2015). On the other hand, 5-FU-1,3,4-selenadiazole conjugate, which was synthetized from 5-FU acid (Fig 5), displayed good activity over kidney-type

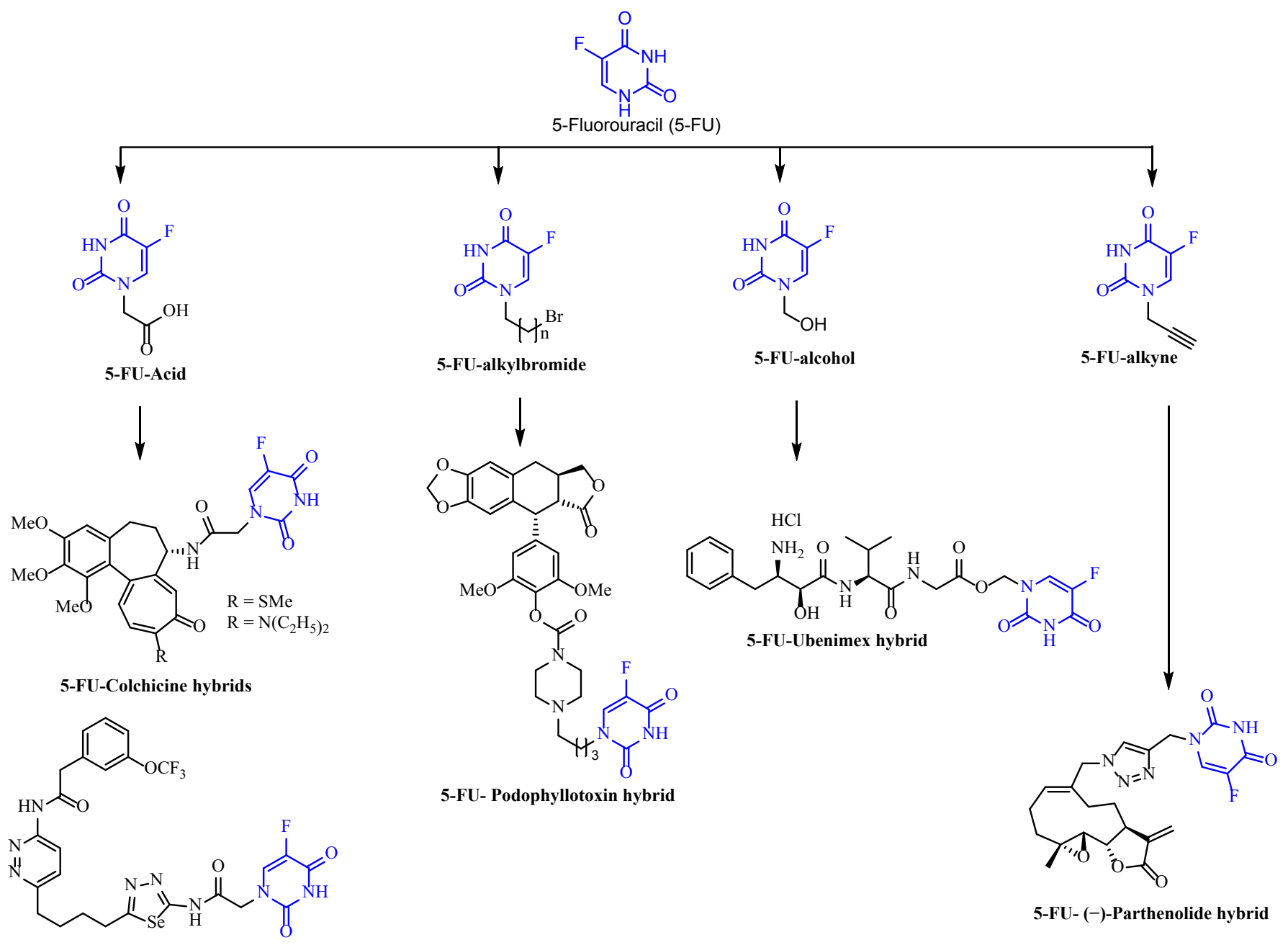

5-FU-1,3,4-Selenadiazole conjugate

Figure 5. Chemical structures of 5-FU precursors, 5-FU conjugates, and 5-FU hybrids. 
glutaminase (KGA) and A549 cell lines with $\mathrm{IC}_{50}$ values of 0.02 and $0.46 \mathrm{uM}$, respectively (Chen et al., 2019).

5-FU alkyl bromide was used as a precursor to the 5-FU podophyllotoxin hybrid (Fig 5). This compound showed high activity with $\mathrm{IC}_{50}$ values of $0.04 \mu \mathrm{M}$ against HL-60 and less than $0.01 \mu \mathrm{M}$ against A-549 (Guan et al., 2016).

FU alcohol was used in the synthesis of 5-FU-Ubenimex hybrid (Fig 5). This hybrid displayed significant in vitro antiproliferation $\left(\mathrm{IC}_{50}=16.52 \mathrm{uM}\right.$ on $\left.\mathrm{PLC} / \mathrm{PRF} / 5\right)$, proapoptosis, antimetastasis, antiangiogenesis, and $\mathrm{CD}_{13}{ }^{+}$cell elimination effects. Moreover, it exhibited further potent in vivo antimetastasis and lifespan extension effects when compared to the approved 5-FU prodrug capecitabine (Jiang et al., 2018).

5-FU-(-)-parthenolide hybrid was obtained by coupling between 5-FU alkyne and (-)-parthenolide azide derivative (Fig 5). This hybrid displayed $\mathrm{IC}_{50}$ values higher than 20 on the two cell lines tested (Bel7402 and Bel-7402/5-FU) (Ding et al., 2019).

\section{Drug resistance of 5-FU-based compounds}

Despite the good behavior of 5-FU hybrids and conjugates against diverse types of cancer, there are studies reporting drug resistance to these molecules. In the case of colorectal cancer and using a 5-FU-resistance cell from HTC8 cells, a high expression of TCF4 and $\beta$-catenin indicated an upregulated Wnt pathway. Additionally, the suppression of checkpoint kinase 1 explained the resistance to 5-FU. Both findings led to conclude that the checkpoint kinase 1 pathway was suppressed by the Wnt pathway in 5-FU-resistant cells (He et al., 2018). Kim et al. (2018) investigated the role of the AKT pathway on drug resistance to 5-FU in SNU-C5/5-FU cells, which is also 5-FU-resistant human colon cancer cells. They reported that the overactivation of the AKT pathway was responsible for resistance to those drugs. Since the findings suggested that the inhibition of this pathway may overcome the resistance to 5-FU, the authors proposed it as a target for cancer treatment (Kim et al., 2018).

As reviewed by Marjaneh et al. (2019), miniRNAs have gained attention as strategies to treat and assess prognosis and reduce 5-FU resistance in colorectal cancer since those miniRNAs have an important role in sensitivity to anticancer drugs and even in tumor resistance induction. Thus, detecting, understanding, and targeting those mechanisms have been explored as a novel therapeutic approach to treat colorectal cancer (Marjaneh et al., 2019). MiniRNAs strategies have been also applied to other cancer types, for example, pancreatic cancer. Wang et al. (2016a) reported upregulated miR-320a in 5-FU-resistant pancreatic cancer cells and that its overexpression contributed to the pathogenesis of this cancer. The authors also found that the restoration of PDCD4 expression could in part reduce the function of miR-320a in pancreatic cancer cells, which at the end (by targeting PDCD4) could be an alternative therapeutic (Wang et al., 2016a). 5-FU has been combined with a natural compound such as andrographolide (a bioactive component of the medicinal plant Andrographis paniculata) as a potential adjunctive treatment for colorectal cancer. Andrographolide/5-FU cotreatment increased the apoptosis level of HCT116/5-FUR cells with a high level of BAX. The authors found that interaction between andrographolide and $\mathrm{BAX}$ prevented BAX degradation, which led to enhancing mitochondria-mediated apoptosis, leading to a reversed 5-FU resistance. On the contrary, BAX silence reduced the 5-FU effect (Wang et al., 2016b).

\section{Delivery systems and molecular simulations of drugs based on 5-FU}

In connection with drug delivery, some nanoparticles have been designed to improve 5-FU loading capacity. In this sense, hollow mesoporous silica nanoparticles have been designed and functionalized using silanes, which led to the insertion of the amine of carboxyl, cyano, and methyl groups onto the nanoparticles. Since amine groups have comparable hydrophilicity but reverse charge to the 5-FU, the presence of this group increased the loading capacity of 5-FU by $28.89 \%$ (She et al., 2015). Lollo et al. (2019) synthesized a lipophilic 5-FU derivative (by using lauric acid). The reaction product (named 5-FU-C12) was encapsulated into lipid nanocapsule and this led probably to increased activity of the 5-FU-C12. Higher cytotoxic effect of 5-FU-C12 (loaded into lipid nanocapsules) on different tumors cell lines [glioma (9L) and human colorectal (HTC-116) cancer cell line] was reported when it was compared with 5-FU-C12 alone or 5-FU (Lollo et al., 2019). Safwat et al. (2016) used gold nanoparticles (GNPs) and two thiol-containing ligands, thioglycolic acid (TGA) and glutathione (GSH), to improve the efficacy of 5-FU. In that study, a 5-FU/ligand molar ratio of 1:1 and 2:1 for TGA-GNPs and GSHGNPs, respectively, led to maximum 5-FU loading (Safwat et al., 2016). 5-FU loading release was $\mathrm{pH}$-dependent, and anticancer effect against colon cancer cells was better for 5-FU/GSH-GNPs when it is compared with free drug.

Molecular simulation approaches have been used to study 5-FU interactions with covalent organic frameworks (COFs) and metal-organic frameworks (MOFs), among others. Safdari et al. (2017) studied the interaction of 5-FU with graphene oxide nanosheet using density functional theory (DFT) and molecular dynamics (MD) simulation. MD simulation results showed that hydrogen bonds among different functional groups on graphene oxide and 5-FU molecules were promoted by increasing the temperature to $400 \mathrm{~K}$ (Safdari et al., 2017). Hashemzadeh and Raissi (2018) used DTF and MD to study the adsorption behavior of 5-FU on the COF surface. The authors reported that, after the adsorption on the COF surface, $5-\mathrm{Fu}-\mathrm{COF}$ is protected from degradation. Additionally, 5-FU showed high stability on the COF surface (Hashemzadeh and Raissi, 2018). Both experimental and MD studies on MOFs for 5-FU release have been also conducted and led to $\mathrm{pH}$-triggered control (Wang et al., 2017). All those studies have been focused on the design of 5-FU delivery vehicles for cancers.

\section{CONCLUSION}

A clear increase in studies related to 5-FU was observed during 2015-2020, with (i) Biochemistry, Genetics, and Molecular Biology; (ii) Chemical Engineering; and (iii) Materials Science as the main areas (excluding chemistry) of this topic. The studies focused mainly on drug delivery, synthesis of anticancer compounds and their activity, molecular simulation, treatment of cancer cells, and drug resistance. China, India, and the United States were the most productive countries on studies related to 5-FU and they also had the strongest collaboration network. 


\section{ACKNOWLEDGMENT}

Funding: The work has been funded by University of Antioquia, MINCIENCIAS, MINEDUCACIÓN, MINCIT and ICETEX, through the Program Ecosistema Científico Cod. FP44842-2112018 Project numbers, 58537.

\section{CONFLICT OF INTEREST}

The authors declare that they have no conflict of interest.

\section{ETHICAL APPROVAL}

This article does not contain any studies with human participants or animals performed by any of the authors.

\section{CONSENT TO PARTICIPATE}

For this type of study, formal consent is not required.

\section{AUTHORS' CONTRIBUTION}

Conceptualization: WCG and HRM; methodology: HRM; formal analysis and investigation: WCG and HRM; writing and original draft preparation: HRM; writing and review and editing: WCG and HRM.

\section{REFERENCES}

Aragón N, Jaramillo-Echeverry A, Ramirez-Malule $\mathrm{H}$. Bibliometric analysis of bacterial resistance on periodontal disease. J Appl Pharm Sci, 2021; 11(04):118-24.

Banerjee A, Pathak S, Subramanium VD, G D, Murugesan R, Verma RS. Strategies for targeted drug delivery in treatment of colon cancer: current trends and future perspectives. Drug Discov Today, 2017; 22(8):1224-32.

Cardona-G W, Herrera-R A, Castrillón-L W, Ramírez-Malule $\mathrm{H}$. Chemistry and anticancer activity of hybrid molecules and derivatives based on 5-Fluorouracil. Curr Med Chem, 2021; 28:1.

Chen Z, Li D, Xu N, Fang J, Yu Y, Hou W, Ruan H, Zhu P, Ma R, Lu S, Cao D, Wu R, Ni M, Zhang W, Su W, Ruan B . Novel 1,3,4-selenadiazole-containing kidney-type glutaminase inhibitors showed improved cellular uptake and antitumor activity. J Med Chem, 2019; 62(2):589-603.

Ding Y, Li S, Ge W, Liu Z, Zhang X, Wang M, Chen T, Chen $\mathrm{Y}$, Zhang Q. Design and synthesis of parthenolide and 5-Fluorouracil conjugates as potential anticancer agents against drug resistant hepatocellular carcinoma. Eur J Med Chem, 2019; 183:111706.

Gómez-Ríos D, Ramírez-Malule H. Bibliometric analysis of recent research on multidrug and antibiotics resistance (2017-2018). J Appl Pharm, Sci. 2019; 9(5):112-16.

Gómez-Ríos D, López-Agudelo VA, Ramírez-Malule H. Repurposing antivirals as potential treatments for SARS-CoV-2: from SARS to COVID-19. J Appl Pharm Sci, 2020; 10(5):1-9.

Guan XW, Xu XH, Feng SL, Tang ZB, Chen SW, Hui L. Synthesis of hybrid 4-deoxypodophyllotoxin-5-fluorouracil compounds that inhibit cellular migration and induce cell cycle arrest. Bioorg Med Chem Lett, 2016; 26(6):1561-6.

Hashemzadeh H, Raissi, H. Covalent organic framework as smart and high efficient carrier for anticancer drug delivery: a DFT calculations and molecular dynamics simulation study. J Phys D Appl Phys, 2018; 51(34):345401.
He L, Zhu H, Zhou S, Wu T, Wu $\mathrm{H}$, Yang $\mathrm{H}$, Mao $\mathrm{H}$, SekharKathera C, Janardhan A, Edick AM, Zhang A, Hu Z, Pan F, Guo Z. Wnt pathway is involved in 5-FU drug resistance of colorectal cancer cells. Exp Mol Med, 2018; 50(8):1-12.

Jiang Y, Li X, Hou J, Huang Y, Wang X, Jia Y, Wang Q, Xu W, Zhang J, Zhang Y. Synthesis and biological characterization of ubenimexfluorouracil conjugates for anti-cancer therapy. Eur J Med Chem, 2018; 143:334-47.

Kim EJ, Kang GJ, Kang JI, Boo HJ, Hyun JW, Koh YS, Chang WY, Kim YR, Kwon JM, Maeng YH, Yoo ES, Lee CH, Kang HK. Overactivation of AKT signaling leading to 5-fluorouracil resistance in SNUC5/5-FU cells. Oncotarget. 2018; 9(28):19911-28.

Liao H, Tang M, Luo L, Li C, Chiclana F, Zeng XJ. A bibliometric analysis and visualization of medical big data research. Sustainability, 2018, 10(2):166.

Lollo G, Matha K, Bocchiardo M, Bejaud J, Marigo I, VirgoneCarlotta A, Dehoux T, Rivière C, Rieu JP, Briançon S, Perrier T, Meyer $\mathrm{O}$, Benoit JP. Drug delivery to tumours using a novel 5-FU derivative encapsulated into lipid nanocapsules. J Drug Target, 2019; 27(5-6):634-45.

Marjaneh RM, Khazaei M, Ferns GA, Avan A, Aghaee-Bakhtiari $\mathrm{SH}$. The role of microRNAs in 5-FU resistance of colorectal cancer: possible mechanisms. J Cell Physiol, 2019; 234(3):2306-16.

Miller KD, Nogueira L, Mariotto AB, Rowland JH, Yabroff KR, Alfano CM, Jemal A, Kramer JL, Siegel RL. Cancer treatment and survivorship statistics, 2019. CA Cancer J Clin, 2019; 69(5):363-85.

Miller KD, Siegel RL, Lin CC, Mariotto AB, Kramer JL, Rowland JH, Stein KD, Alteri R, Jemal A. Cancer treatment and survivorship statistics, 2016. CA Cancer J Clin, 2016; 66(4):271-89.

Nevedomskaya E, Baumgart SJ, Haendler B. Recent advances in prostate cancer treatment and drug discovery. Int J Mol Sci, 2018; 19(5):1359.

Ramirez-Malule H. Bibliometric analysis of global research on clavulanic acid. Antibiotics, 2018; 7(4):102

Ramírez-Malule H, López-Agudelo VA, Gómez-Ríos D. Candida auris: a bibliometric analysis of the first ten years of research (2009-2018). J Appl Pharm Sci, 2020; 10(3):12-21.

Ramírez-Malule H, Quiñones-Murillo DH, Manotas-Duque D. Emerging contaminants as global environmental hazards. A bibliometric analysis. Emerg Contam, 2020; 6:179-93.

Rejhová A, Opattová A, Čumová A, Slíva D, Vodička P. Natural compounds and combination therapy in colorectal cancer treatment. Eur J Med Chem, 2018; 144:582-94.

Ribeiro RA, Wanderley CW, Wong DV, Mota JM, Leite CA, Souza MH, Cunha FQ, Lima-Júnior RC. Irinotecan- and 5-fluorouracilinduced intestinal mucositis: insights into pathogenesis and therapeutic perspectives. Cancer Chemother Pharmacol, 2016; 78(5):881-93.

Safdari F, Raissi H, Shahabi M, Zaboli M. DFT calculations and molecular dynamics simulation study on the adsorption of 5-fluorouracil anticancer drug on graphene oxide nanosheet as a drug delivery vehicle. J Inorg Organomet Polym Mater, 2017; 27(3):805-17.

Safwat MA, Soliman GM, Sayed D, Attia MA. Gold nanoparticles enhance 5-fluorouracil anticancer efficacy against colorectal cancer cells. Int J Pharm, 2016; 513(1-2):648-58.

Sara JD, Kaur J, Khodadadi R, Rehman M, Lobo R, Chakrabarti S, Herrmann J, Lerman A, Grothey A. 5-Fluorouracil and cardiotoxicity: a review. Ther Adv Med Oncol, 2018: 10:175883591878014.

She X, Chen L, Li C, He C, He L, Kong L. Functionalization of hollow mesoporous silica nanoparticles for improved 5-FU loading. J Nanomater, 2015; 2015:1-9.

Shen L, Hu J, Wang H, Wang A, Lai Y, Kang Y. Synthesis and biological evaluation of novel uracil and 5-Fluorouracil-1-Yl acetic acidcolchicine conjugate. Chem Res Chinese Univ, 2015; 31(3):367-71.

Soosaraei M, Khasseh AA, Fakhar M, Hezarjaribi HZ. A decade bbliometric analysis of global research on leishmaniasis in web of science database. Ann Med Surg, 2018; 26:30-7. 
Sung H, Ferlay J, Siegel RL, Laversanne M, Soerjomataram I, Jemal A, Bray F. Global cancer statistics 2020: GLOBOCAN estimates of incidence and mortality worldwide for 36 cancers in 185 Countries. CA Cancer J Clin, 2021; 71(3):201-49.

Tecza K, Pamula-Pilat J, Lanuszewska J, Butkiewicz D, Grzybowska E. Pharmacogenetics of toxicity of 5-fluorouracil, doxorubicin and cyclophosphamide chemotherapy in breast cancer patients. Oncotarget, 2018;9(10):9114-9136.

Waks AG, Winer EP. Breast cancer treatment: a review. JAMA, 2019; 321(3):288-300.

Wang FM, Wang J, Yang SZ, Gu CY, Wu XR, Liu JQ, Sakiyama $\mathrm{H}, \mathrm{Xu}$ JW, Luo MM, Liu WC. A combination of experiment and molecular simulation studies on a new metal-organic framework showing PH-triggered drug release. J Coord Chem, 2017; 43(2):133-7.

Wang W, Zhao L, Wei X, Wang L, Liu S, Yang Y, Wang F, Sun G, Zhang J, Ma Y, Zhao Y, Yu J. MicroRNA-320a promotes 5-FU resistance in human pancreatic cancer cells. Sci Rep, 2016a; 6(1):27641.
Wang W, Guo W, Li L, Fu Z, Liu W, Gao J, Shu Y, Xu Q, Sun Y, $\mathrm{Gu}$ Y. Andrographolide reversed 5-FU resistance in human colorectal cancer by elevating BAX expression. Biochem Pharmacol, 2016b; 121:8-17.

WHO. Cancer. WHO, Geneva, Switzerland. Available via https://www.who.int/news-room/fact-sheets/detail/cancer (Accessed 28 March 2021)

How to cite this article:

Ramirez-Malule H, Cardona-G W. Bibliometric analysis of recent research on 5-Fluorouracil (2015-2020). J Appl Pharm Sci, 2022; 12(01):070-077. 